Rabaska

Revue d'ethnologie de l'Amérique française

\title{
Le patrimoine religieux du Québec : de l'objet cultuel à l'objet culturel. Retour sur un colloque
}

\section{Julie-Éliane Beaulieu}

Volume 3, 2005

URI : https://id.erudit.org/iderudit/201717ar

DOI : https://doi.org/10.7202/201717ar

Aller au sommaire du numéro

Éditeur(s)

Société québécoise d'ethnologie

ISSN

1703-7433 (imprimé)

1916-7350 (numérique)

Découvrir la revue

Citer ce document

Beaulieu, J.-É. (2005). Le patrimoine religieux du Québec : de l'objet cultuel à

l'objet culturel. Retour sur un colloque. Rabaska, 3, 124-127.

https://doi.org/10.7202/201717ar d'utilisation que vous pouvez consulter en ligne. 


\title{
Colloque
}

\section{Le patrimoine religieux du Québec : de l'objet cultuel à l'objet culturel. \\ Retour sur un colloque}

\author{
Julie-Éliane Beaulieu
}

Université Laval

L'avenir du patrimoine religieux suscite des réactions multiples et parfois contradictoires et ce, dans plusieurs pays. Le Québec fait également face à une problématique aux facteurs pluriels en ce qui a trait à son patrimoine religieux. Les communautés religieuses sont vieillissantes et la relève est faible, la fréquentation des églises connaît une baisse considérable et les moyens financiers manquent pour assurer l'entretien des bâtiments. Ces constats impliquent une réflexion et des prises de décision qui concernent toute la société. Le passage « de l'objet cultuel à l'objet culturel » énonce bien l'idée inspiratrice du colloque international qui s'est déroulé du 12 au 14 novembre 2004 en l'église Saint-Dominique de la Grande-Allée, à Québec.

Organisé par la Chaire de recherche du Canada en patrimoine ethnologique, dont le titulaire est Laurier Turgeon, professeur à l'Université Laval, ce grand colloque a réuni environ 350 participants intéressés aux questions débattues par les intervenants des six séances au programme.

Les «jeux et les enjeux » (séance 1) actuels sont de la taille d'une cathédrale. Le patrimoine religieux immobilier n'est pas le seul bien à considérer; il faut y ajouter les objets de culte, mais aussi les savoirs et les savoir-faire reliés aux pratiques religieuses. Le colloque visait cet objectif d'une réflexion et d'un partage des idées et des connaissances en ce qui a trait à la sauvegarde, à la conservation (séance 3), à la connaissance par la recherche, mais également à la reconnaissance par la diffusion et la mise en valeur de la richesse de ces différents ensembles, immobiliers, mobiliers et humains (séances 4, 5 et 6). Pour un colloque, à caractère international, seuls quelques conférenciers français sur les quarante-deux participants présentèrent des allocutions aux côtés de leurs confrères québécois. Aussi, bien que le patrimoine religieux du Québec soit principalement catholique, il aurait été enrichissant d'élargir davantage le champ d'intervention à d'autres 
confessions religieuses afin d'élargir les horizons de nos réflexions. Néanmoins, la religion protestante fit l'objet de trois communications.

Ce compte rendu s'intéresse exclusivement à la deuxième séance qui abordait le thème central du colloque, soit le passage « de l'objet cultuel à l'objet culturel ». Des six intervenants, trois provenaient d'institutions muséales. John R. Porter est le directeur du Musée national des beaux-arts du Québec, Christine Turgeon, la directrice du Musée des Ursulines de Québec, et Marcel Daneau, le directeur du Musée des religions de Nicolet. Pour sa part, Jean-Yves Andrieux est professeur des Universités à Rennes et Philippe Chareyre est professeur à l'Université de Pau et des Pays de l'Adour dans le sud-ouest de la France. Quant à Richard Gauthier, il est consultant en urbanisme pour la ville de Montréal.

Monsieur John R. Porter rappela le rôle et l'engagement que joue le MNBAQ en matière de patrimoine religieux et ce, depuis sa fondation en 1933. Le Musée a présenté plusieurs expositions à caractère religieux, tel « Le Grand Héritage » en 1984. Le Musée est un conseiller et un partenaire pour les communautés, l'exposition sur les broderies des Ursulines et «Copyright Rubens » en association avec les Augustines en témoignent. Cette volonté de diffusion et de transmission s'inscrit dans la mission éducative d'un Musée d'état. Le passage du cultuel au culturel est réalisé avec tout le respect nécessaire du caractère sacré des objets. Pour reprendre l'idée de Monsieur Dominique Ponnau, il est important de connaitre la valeur cultuelle d'un objet et celle-ci ne doit pas être dissociée de la valeur culturelle si l'on veut en saisir le sens. Le directeur du MNBAQ insista aussi sur l'importance de la conservation et de la restauration minutieuse et professionnelle des œuvres, des priorités pour le Musée. Il favorise par la recherche « la connaissance pour la reconnaissance et la prise en main de notre patrimoine religieux $»$. L'effet de désacralisation suscite moins d'émotion lorsque les objets entrent dans un musée de notoriété. Et pourtant...

Le Musée des Ursulines est un bel exemple de réussite dans l'apport du cultuel au culturel. Christine Turgeon dresse l'évolution du musée de l'institution fondée en 1639 et dont le patrimoine in situ est d'une grande valeur. Ce qui fait la richesse du Musée, c'est justement son identité physique et mémorielle, qui permet aux visiteurs de connaître et de comprendre l'histoire des Ursulines de manière globale, tant au plan de la vie conventuelle que de sa mission éducative. Le Musée fait montre des initiatives de la communauté pour faire connaître et apprécier ce qui fait partie de l'histoire collective et, en ce sens, les Ursulines sont « des passeuses du patrimoine », pour employer les termes de Madame Turgeon. La congrégation a su conserver au fil des siècles ses archives mais également des objets qui témoignent des savoirs et des savoir-faire ainsi que de la vie quotidienne dans le couvent. La 
richesse patrimoniale dépasse l'aspect religieux, elle s'insère dans la société. Le cultuel est passé au culturel par l'entreprise même des Ursulines.

Le Musée des religions de Nicolet a pour mission de faire connaître les religions par le biais d'objets souvent banals, mais porteurs de mémoire. Ce Musée à caractère ethnologique a constitué sa collection, composée en grande partie d'objets catholiques, principalement à partir de dons. Le Musée représente un agent unique et précieux pour la préservation d'une partie souvent méprisée du patrimoine religieux, mais dont la richesse symbolique peut enrichir nos connaissances. Tout comme son confrère du MNBAQ, Monsieur Daneau veut protéger et transmettre ce patrimoine. La méconnaissance des jeunes générations face à l'histoire religieuse mérite une attention particulière et, dans ce sens, les trois Musées cités ont à cœur leur mission éducative.

Le quatrième exposé, celui de Jean-Yves Andrieux, porte sur un joyau du patrimoine de France, l'abbaye de Sénanque dans le Vaucluse. Depuis sa fondation par les cisterciens en 1148 jusqu'à aujourd'hui, ce lieu d'une grande richesse patrimoniale passera du cultuel au culturel pour revenir au cultuel dans les années 1980. Les travaux réalisés par les locataires de la société lyonnaise des Automobiles Marius Berliet, à partir de 1969, témoigne d'un souci de valorisation notable et bénéfique. L'endroit devient un musée du Sahara et sert pour différentes expositions, concerts et colloques. L'abbaye retrouve finalement sa fonction religieuse en 1988, tout en conservant ses attraits touristiques et historiques. L'abbaye de Sénanque est un exemple à considérer dans la conjoncture actuelle en ce qui a trait au patrimoine religieux. Il faut garder en mémoire qu'un lieu de culte abandonné, et pour lequel on trouve une nouvelle utilisation, pourrait un jour réintégrer sa fonction première. Dans cette optique, la décision de recycler certains bâtiments doit tenir compte dans les évaluations des possibilités de reconversion. À titre d'exemple, l'église Saint-Matthew, qui abrite la Bibliothèque du quartier Saint-Jean-Baptiste peut facilement recouvrer ses activités liturgiques en cas de besoin.

Ce dernier constat nous amène à l'allocution de Monsieur Gauthier sur le « recyclage » de certaines églises, notamment celles qui ont connu « une approche à rebours ». Une " gestion économique du vide » se traduit par l'utilisation de la structure d'un bâtiment comme c'est le cas pour l'église Notre-Dame-de-Foy ou celle de Saint-Boniface. Richard Gauthier maintient l'idée que le sentiment de perte est compensé par la sauvegarde du squelette que l'on convertit à moindre coût en jardin. Il propose le jeu et l'audace d'une appropriation différente des lieux de culte. Son projet de « coulée verte » dans la ville de Montréal, qui passerait dans une église dont le côté serait préalablement ouvert sur l'extérieur, risque fort de ne pas faire l'unanimité 
chez les congrégations religieuses. Il y a une différence entre l'utilisation d'une structure restée debout après un incendie et une église complète. Néanmoins, l'idée semble intéressante dans le cas de certaines églises et ce type de projet écologique permettrait une réappropriation citoyenne des lieux.

La dernière présentation de la séance concerne les protestants béarnais et l'avenir de son patrimoine. Philippe Chareyre s'interroge sur la minorité protestante du Béarn (pays de naissance d'Henri IV) qui possède un musée de l'histoire du protestantisme ainsi qu'un centre d'étude. La volonté d'amasser des témoignages matériels afin de constituer un fonds pour la recherche historique est à coup sûr garant de cette mémoire. Par contre, le mobilier se fait rare chez les protestants et les temples sont peu nombreux, mais les archives sont heureusement abondantes. Pour la sauvegarde de ce patrimoine fragile, plusieurs actions ont été pensées. Des campagnes de filmographie de la liturgie sont menées, les résultats des recherches sont publiés et le musée qui se trouve dans la maison de Jeanne d'Albret, mère d'Henri IV ( $\mathrm{XVI}^{\mathrm{e}}$ siècle), présente des maquettes, à défaut d'objets. Une campagne de filmographie est un moyen peu coûteux et utile pour recueillir le patrimoine immatériel des communautés. Comme le mentionne Diane Audy, l'immatériel est le code d'accès pour notre compréhension et pour rattacher des histoires et des émotions aux objets.

Il ne semble pas y avoir de passage de l'objet cultuel à l'objet culturel, mais plutôt un va-et-vient constant entre les deux. On a démontré le rôle essentiel de plusieurs types de musées en matière de patrimoine religieux. Le Musée Marguerite-Bourgeoys à Montréal est un autre exemple de l'importance de développer des musées ou des centres d'interprétation in situ. La coulée verte s'inscrit également dans le processus de revitalisation. Un tel projet traversant une église lui redonnerait sa fonction d'accueil et pourrait servir de lieu de concert durant l'été. Le patrimoine immatériel est le plus difficile à saisir et, face aux communautés religieuses vieillissantes, il $\mathrm{y}$ a une urgence de cueillette. Il faut penser à inclure un volet interculturel dans une telle entreprise.

Les institutions muséales et religieuses ne peuvent à elles seules assurer la protection du patrimoine religieux. L'éducation peut former une connaissance et une conscience pour une protection citoyenne. La problématique doit être prise dans son ensemble, bien que chaque cas soit unique et mérite que l'on s'y attarde afin de prendre la meilleure décision. Le gouvernement doit au moins assurer son rôle législatif s'il ne peut plus financer les projets d'envergure. En terminant, il faut rappeler que, suite à ce colloque, le plus important maintenant est de faire des choix et de poser des actions. Comme le disait Mario Dufour au sujet du projet du Monastère des Augustines de l'Hôtel-Dieu à Québec : «Faisons-le et ça se fera! ». 